\title{
Simulation of gas pipeline leakage using the characteristics method
}

\author{
E. Nourollahi \\ NIGC (National Iranian Gas Company), \\ Department of Mechanical Engineering, Ferdowsi University, Iran
}

\begin{abstract}
The modeling of gas leakage for understanding the process of pressure reduction and gas leakage rate of a hole is accomplished by zero-dimensional models of the tank and pipe. These models of the tank and pipe are suitable for simulation when the hole is very small or where there is a complete fracture. However, use of these two methods or a combination of them is suitable rather when the pipelines are very long. In zero-dimensional models the effects of complex boundaries are also ignored. In this study, the pipeline leakage is simulated by use of the one dimensional characteristics method. This model is perfect compared with both pipe or tank models, or a combination of them, and we can expand the simulation of gas pipeline leakage to the short pipelines and variable boundary conditions. This is important in order to calculate the volume of gas discharged from the pipelines when an accident occurs.
\end{abstract}

Keywords: leakage, gas pipeline, characteristics method.

\section{Introduction}

Natural gas is considered as a clean source of energy worldwide. One of the usual problems is finding methods for prevention of wasting natural gas during transportation and distribution. The method that is presented in this paper can result in somewhat subtle calculations when a hole is creating in the pipeline surface and consequently has an important role in the estimate of the received losses.

Pipe surface leakage or pipe section dismissal can be due to various reasons, such as corrosion, earthquake or mechanical stroke, which may be implemented in the pipe surface, and also overload compressors. 
After the leakage has been created, the flat expansion pressure waves are propagated in two converse sides. These waves have sonic speed and after clashing to the upstream and downstream boundaries, return to the form of compression or expansion wave depending on the edge type (Fig. 1). In the leak location, depending whether the ratio of pressure to ambient pressure is more or less than the CPR quantity, the equation of which is showed in equation (1), the flow will be sonic and ultrasonic or subsonic respectively.

$$
C P R=\frac{P_{\text {out }}}{P_{1 c r}}=\left(\frac{2}{k+1}\right)^{\frac{k}{k-1}}
$$

In the above equation, $P_{1 c r}$ is the critical pressure of point 1 and $\mathrm{k}$ is the thermal capacity ratio.

Therefore, the problem physics change to one of the two stated cases below.

If the flow is sonic and ultra sonic, the sonic reporter wave does not leak from out of the pipe to enter the pipe. Hence, the changes of the flow field are accomplished due to the flat pressure waves and the real boundary conditions on the start and end of the pipe. The mass flow outlet of the hole also depends only on the stagnation pressure in the leak location and on the area of the hole and is not related to the form of the orifice cross section.

However, if the flow in the leak location is subsonic, the hole could be the source of the production of a compression or expansion sonic wave and the effect on the flow field. In this situation, the pipe and leakage spot act as a $\mathrm{T}$ junction and the mass outflow of the leak location depends on the ambient pressure and a coefficient known as the empirical discharge coefficient in addition to the stagnation pressure and the orifice area.

The pressure with respect to the ambient pressure in the gas transition pipelines are good, the ratio is more than CPR and the gas flow is also provided from the entering sources, such as the permanent gas refinery, hence in this paper the problem physics is considered to be like the state in eqn. (1).

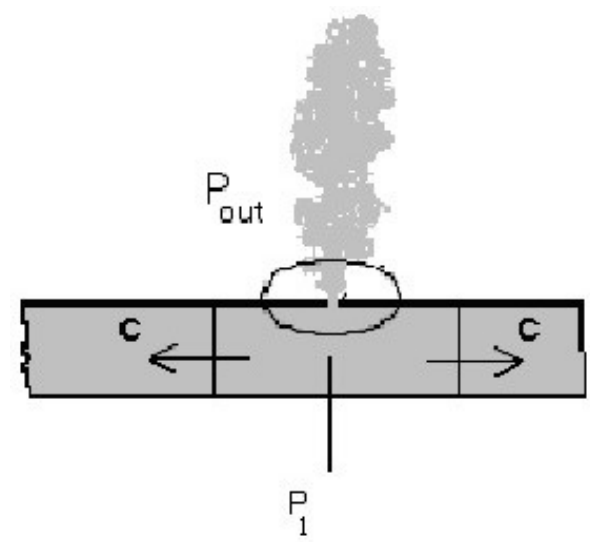

Figure 1: $\quad$ Leakage display and the waves that are produced. 
According to what has been stated about problem physics (which means that changes in the solution field only occur by the flat pressure wave), the solution of gas flow in a leaking pipe can be suitable for use of the one dimensional numerical method. Therefore, in this paper is the one dimensional characteristics method is used for modeling the compressible gas flow in the pipe and for calculation of the leakage flow. The assumption is that the inner flow of the pipe is homentropic; hence the solution of the mass and momentum conservation addition to the constant entropy flow assumption are sufficient for flow simulation. In continuance the characteristics method is introduced, then the necessary correction is stated for considering the leakage effect on the flow field in order to determine the leakage flow and the pressure distribution in the pipe line.

\section{Characteristics method}

Homentropic flow (a flow that has the same entropy level in the whole field) is a special case of the general flow problems and its solution is fairly straightforward compared with the more complicated general flow problems. In this part the method of characteristics is introduced to solve this problem $[1,2]$.

The equations of mass and momentum conservation, without considering friction, are the forms below.

The continuity equation is:

$$
\frac{\partial(\rho u)}{\partial x}=-\frac{\partial \rho}{\partial t}
$$

The momentum equation is:

$$
-\frac{\partial P}{\partial x}=\rho \frac{D u}{D t}
$$

In these equations $\rho$ is gas density and $u$ is the speed of the gas.

With attention to the definition of the speed of the sound $a$ by:

$$
a^{2}=\left(\frac{\partial p}{\partial \rho}\right)_{s}
$$

For an ideal gas:

$$
a^{2}=\frac{k p}{\rho}
$$

By using of the relationship between the sonic speed and the pressure in an ideal gas, these equations are changed to the below forms after some steps of rewriting of the mass and momentum conservation equations:

$$
\left\{\frac{\partial a}{\partial t}+(u+a) \frac{\partial a}{\partial x}\right\}+\frac{k-1}{2}\left\{\frac{\partial u}{\partial t}+(u+a) \frac{\partial u}{\partial x}\right\}=0
$$




$$
\left\{\frac{\partial a}{\partial t}+(u-a) \frac{\partial a}{\partial x}\right\}-\frac{k-1}{2}\left\{\frac{\partial u}{\partial t}+(u-a) \frac{\partial u}{\partial x}\right\}=0
$$

Equations (6) and (7) form a set of quasi-linear hyperbolic partial differential equations. A solution of the form:

is required.

$$
\begin{aligned}
& a=a(x, t) \\
& u=u(x, t)
\end{aligned}
$$

In order to obtain a characteristic solution it is assumed that $a$ and $u$ are uniquely related by this expression:

$$
c=c(u, a)
$$

Then, the solution to eqns. (6) and (7) will be of the form:

$$
c=c(x, t)
$$

The solution may be represented by the curved surface bounded by, say, the edges PQRS as shown in Fig. 2.

According to the definition, if in a special point on the surface of $c=c(x, t)$ for a reviewing special curve from that point, the slope of the projected curve on the $x$-t plane is equal with the quantity of the curve of that point, the passing direction of that point is known as the characteristic direction. We have in mathematical expression:

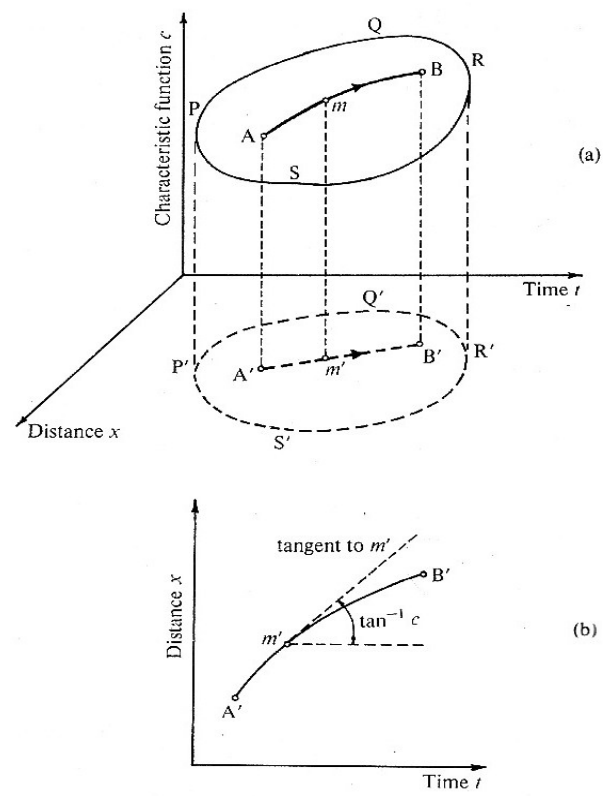

Figure 2: Graphical interpretation of the characteristics method. (a) Threedimensional surface defining $c=c(x, t)$. (b) Projection of line on the characteristics surface to the plane at $c=0$. 


$$
\mathrm{C}=(d x / d t)
$$

By using this complete derivative definition, the sonic speed and particle speed parameters are determined with respect to the time of a characteristic length, such as the below:

$$
\begin{aligned}
& \left(\frac{d a}{d t}\right)_{\text {char }}=\frac{\partial a}{\partial t}+c \frac{\partial a}{\partial x} \\
& \left(\frac{d u}{d t}\right)_{\text {char }}=\frac{\partial u}{\partial t}+c \frac{\partial u}{\partial x}
\end{aligned}
$$

Therefore if $c=c(u, a)$ is defined as the form of $\begin{array}{r}c_{1}=u+a \\ c_{2}=u-a\end{array}$, eqns. (6) and (7) in length of two characteristics $c_{1}$ and $c_{2}$ are rewritten like this:

$$
\begin{aligned}
& \left(\frac{d a}{d t}\right)_{c 1}+\frac{k-1}{2}\left(\frac{d u}{d t}\right)_{c 1}=0 \\
& \left(\frac{d a}{d t}\right)_{c 2}-\frac{k-1}{2}\left(\frac{d u}{d t}\right)_{c 2}=0
\end{aligned}
$$

Consequently a total solution is always calculable by use of the below characteristic equations and also by use of the numerical or graphic methods.

$$
\begin{aligned}
& \frac{d x}{d t}=u \pm a \\
& \frac{d a}{d u}=\mp \frac{k-1}{2}
\end{aligned}
$$

Hence, the solution of these momentum and mass conservation equations in any time step should be determined two characteristics:

$$
c_{1}=u+a \text { and } c_{2}=u+a
$$

\subsection{The numerical solution method}

At first, the non-dimensional parameters of $\mathrm{A}$ and $\mathrm{U}$ are defined as below in the characteristics method:

$$
U=\frac{u}{a_{r e f}} \quad ; \quad A=\frac{a}{a_{r e f}}
$$

In the above equation $a_{r e f}$ is the sonic speed at the start point. Then, the Reimann non-dimensional characteristics are defined as follows:

$$
\lambda=A+\frac{k-1}{2} U \quad ; \quad \beta=A-\frac{k-1}{2} U
$$

Therefore, determination of the two parameters of $\beta, \lambda$ in any point of the solution field is going to bring about the speed and pressure at that point. 
An explicit equation between Reimann variables in the inner points of the solution field is presented below, which is for each step:

$$
\begin{aligned}
& \lambda_{i}^{n+1}=\lambda_{i}^{n} \frac{\Delta t}{\Delta x}\left\{b \lambda_{i-1}^{n}-a \beta_{i-1}^{n}\right\}\left\{\lambda_{i-1}^{n}-\lambda_{i}^{n}\right\} \\
& \beta_{i}^{n+1}=\beta_{i}^{n} \frac{\Delta t}{\Delta x}\left\{b \beta_{i+1}^{n}-a \lambda_{i+1}^{n}\right\}\left\{\beta_{i+1}^{n}-\beta_{i}^{n}\right\}
\end{aligned}
$$

For determination of the relation between the Reimann variables in boundary points, it should be known that the boundary condition means the equation of state, which is determining for the relation between $a$ and $u$. Therefore, various forms of boundary conditions can be introduced for numerical solution of the problem. These boundary conditions can be open start or open end, close start or close end, the start contact to an entering valve with the special opening percentage, the end contact to an exit nozzle with special opening percentage, the related end to a valve that has definite pressure drop, the start contact to a reservoir, the end without the changes with respect to the location, the close start and close end or the other boundary conditions.

By distinction of the state equation in the boundary, a mono-equation is created between Reimann variables $\beta, \lambda$ and so always in any boundary, one of these variables is known and the other one is unknown, then the unknown Reimann variable can be calculated, so the effect of the boundary transfers to the solution field is obtained.

\subsection{The implementation of the leakage effect}

For implementation of the leakage effect on the flow field, the mesh is chosen in a way that the hole location would be stated between two nodes, a in Figure 3.

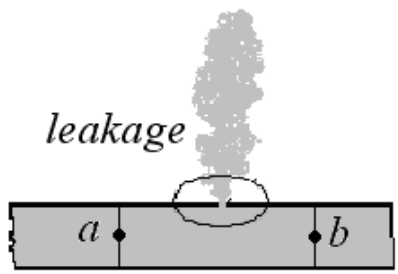

Figure 3: Location of the hole between two nodes.

When the hole is created in the pipe surface, as is said, the pressure ratio to the ambient pressure below the hole, which is in the pipe, is more than the CPR in the later time steps. Therefore, the flow is checked in the hole location and the outflow of the leak location is calculated by the equation below [3]:

$$
Q=A_{o r} P_{l} \cdot \sqrt{\frac{M}{Z R T_{l}} \cdot k \cdot\left(\frac{2}{k+1}\right)^{\frac{k+1}{k-1}}}
$$


In the above equation, $A_{o r}$ is the hole (orifice) area, $\mathrm{M}$ is the molecular weight, $\mathrm{Z}$ is the compressibility factor, $\mathrm{R}$ is the constant of gas and $T_{1}$ is the temperature of gas in below the hole.

The leakage point in any time step acts as a boundary and two expansion waves, depending on the direction of the flow in the pipe, would reach the $a$ and $b$ points with little time difference and create the same change in the nondimensional speed of $U$, as in the form below:

$$
\begin{aligned}
& U_{a}=U_{a}-\frac{Q}{\dot{m}_{a}} \cdot U_{a} \\
& U_{b}=U_{b}+\frac{Q}{\dot{m}_{b}} \cdot U_{b}
\end{aligned}
$$

Therefore, two known characteristics, such as $\lambda_{a}$ and $\beta_{b}$, according to the definition of the positive direction (towards boundary) for the particle speed are corrected like this:

$$
\begin{aligned}
& \lambda_{a}=\frac{\left(2 * A_{a}+(k-1) * U_{a}\right)}{2} \\
& \beta_{b}=\frac{\left(2 * A_{b}-(k-1) * U_{b}\right)}{2}
\end{aligned}
$$

Then the unknown parameters $\lambda_{b}$ and $\beta_{a}$ are calculated as in the form below:

$$
\begin{aligned}
& \beta_{a}=\lambda_{a}-(k-1) * U_{a} \\
& \lambda_{b}=\beta_{b}-(k-1) * U_{b}
\end{aligned}
$$

Therefore, the state of two points $a$ and $b$ in any time step, considering the corrected leakage effect and hence by notice to the equations that govern the problem, being hyperbolic types of equations, during the time of the leakage, the effect is transferred permanently as a third boundary addition to the upstream and downstream boundaries to the solution field.

\section{Results of numerical solution}

In this part, the results of the simulation of the leakage are presented for a pipe which of 250 meter in length and with a hole (orifice) of $1 \mathrm{~cm}^{2}$ area on the surface, by using a grid system with 100 nodes. It is also considered that the hole is in the middle of the pipe length and it is assumed that the initial gas pressure and initial gas speed are $30 \mathrm{bar}$ and $41 \mathrm{ft} / \mathrm{s}$, respectively [4]. It is considered that the upstream boundary condition is the reservoir with constant pressure and the downstream boundary condition is stated with three forms:

- The boundary with no changes with respect to the location.

- The valve with constant coefficient of pressure drop.

- Close end. 
The first situation is when the pipe length is very long compared to the hole, where gas pipelines are like this in most of the cases. The second situation is when the leakage occurred before a valve with the constant pressure drop in a part of the pipe. The third situation is when the pipe end is closed (e.g. when the exit valve is closed completely).

In continuing, the respective results and discussions for the pressure changes at the beginning (because of the propagation of the expansion wave from the leak location) and changes of mass flux by time are presented.

\section{Conclusions}

The related diagrams are shown as mentioned before, for the entering boundary of the constant pressure reservoir and three downstream boundaries, which are described here.

Figures 4 and 5 are for the non-gradient boundary (with respect to the location), Figures 6 and 7 are for the downstream boundary related to the valve with the constant pressure drop and Figures 8 and 9 are for the close downstream boundary. By studying these situations the below conclusions can be inferred.

According to Figures 4(a), 6(a) and 8(a), we observe that the pressure changes on the primary times and the reaching time to the hole; it can be seen that after passing the hole, pressure returns to nearly the first state. We have the pressure increase only in the state of the close downstream boundary condition, due to the constant entering flow. It is also observed that two weak and strong waves are propagated depending on the flow direction. Flow direction in this study is assumed from left to right, then the expansion wave, which moves from the center to the right, is stronger.

By attention to Figures 4(b), 6(b) and 8(b), it is seen that the exit mass flux from the leakage location have an intense fluctuant behavior. The boundary conditions in upstream and downstream also affect the time average of the exit mass flux and the amplitude and frequency of the fluctuations. The average at the first type of boundary condition is about $118 \mathrm{~kg} / \mathrm{s}$, at the second state it is about $110 \mathrm{~kg} / \mathrm{s}$ after 70 seconds and at the third boundary state this average reaches $350 \mathrm{~kg} / \mathrm{s}$.

In the Figures 5(a), 7(a) and 9(a) the quantity of the exit mass flux changes are shown by increasing the hole area and for various lengths of the pipe. It is clear that by increasing the hole area, the quantity of exit mass flux is increased for all the lengths and this subject is true for all three states. By attention to these diagrams, it is also observable that the gradient of the diagram is zero on the long lengths gradually. In other words, the increase of the hole area does not have any effect on the exit mass flux, which is more obvious in figure 5(a).

In Figures 5(b), 7(b) and 9(b), the exit mass flux changes are shown by the pressure and for various lengths of pipes. It is seen that the exit flux quantity is increased for all lengths by increasing the pressure of the pipeline, but the manner of the increase with respect to the various lengths depends on the boundary conditions. So, at the first boundary condition (Figure 5(b)) the increase of the pipe length does not have any effect and the gradient of all the 


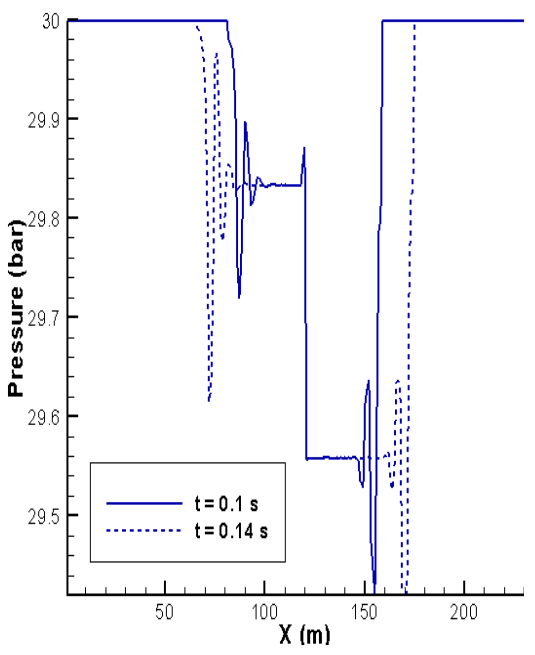

(a)

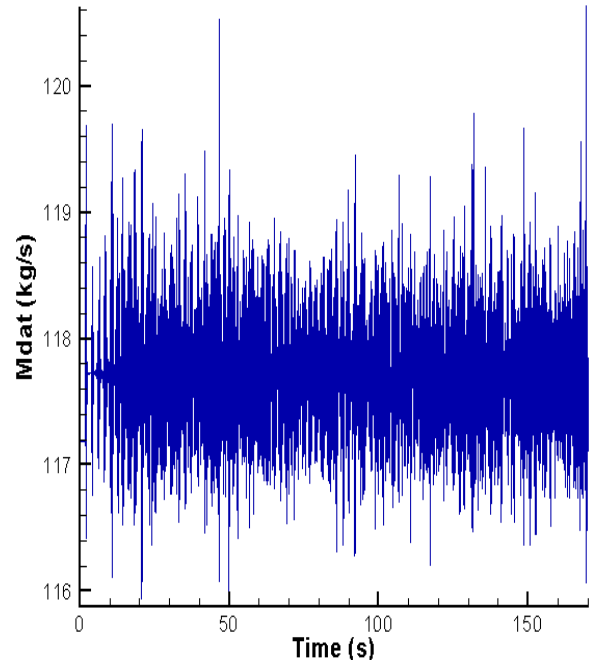

(b)

Figure 4: $\quad$ State (1) of the boundary conditions. (a) Pressure changes by increasing the pipe length at primary times. (b) Changes to the exit mass flux by time.

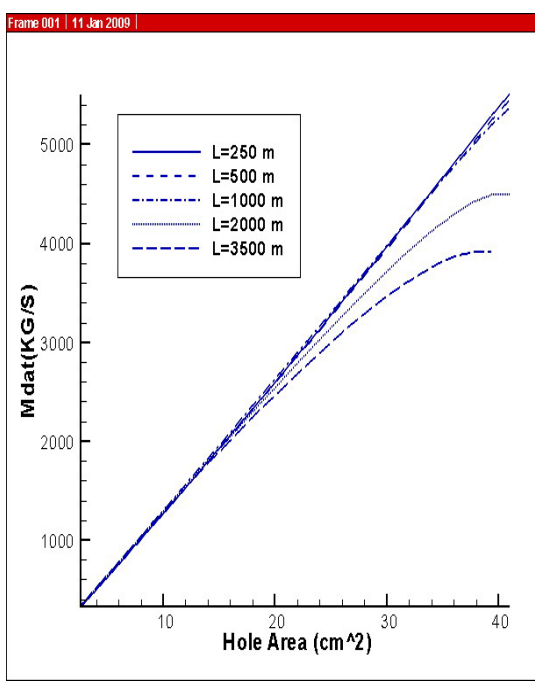

(a)

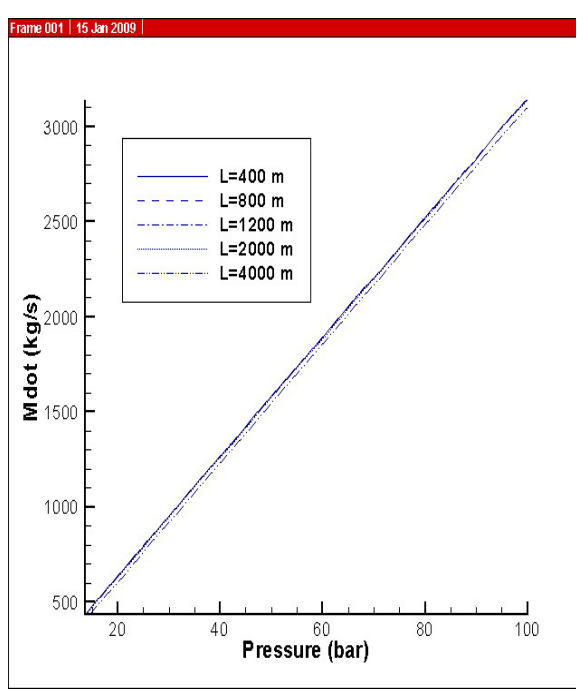

(b)

Figure 5: $\quad$ State (1) of the boundary conditions. (a) Changes to the exit mass flux by increasing the hole area and pipe length. (b) Changes to the exit mass flux by increasing the pipe pressure and pipe length. 


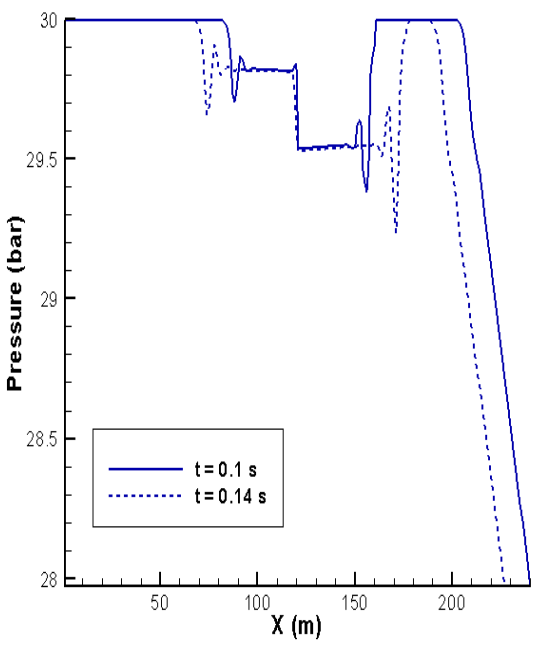

(a)

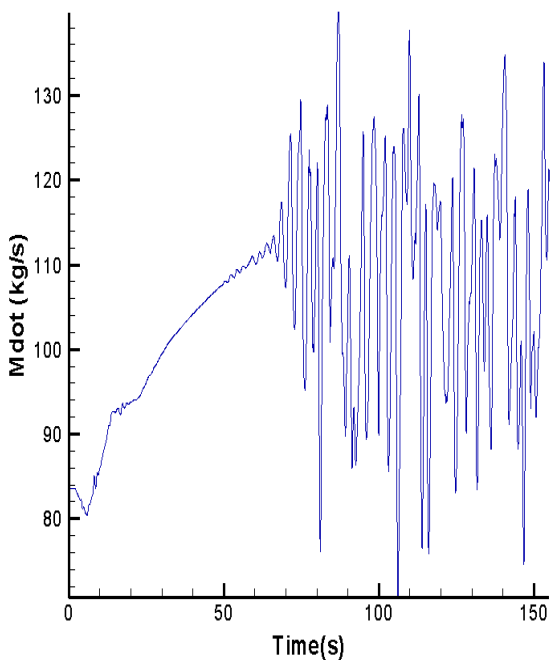

(b)

Figure 6: State (2) of the boundary conditions. (a) Pressure changes by increasing the pipe length at primary times. (b) Changes to the exit mass flux by time.

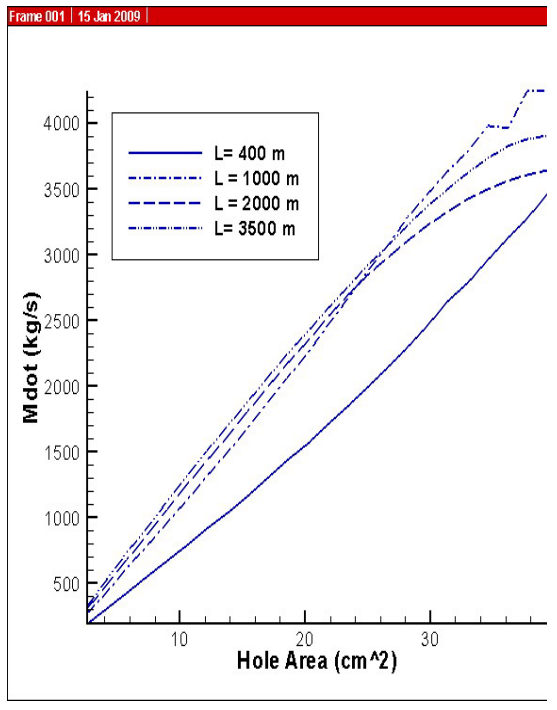

(a)

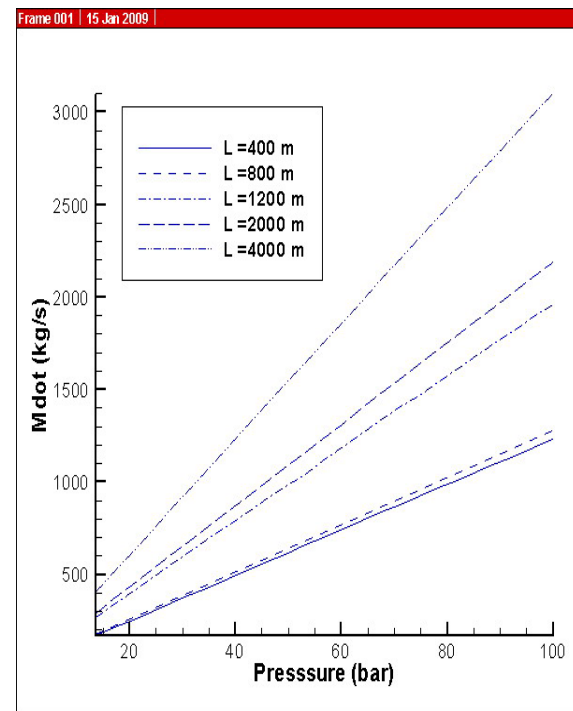

(b)

Figure 7: $\quad$ State (2) of the boundary conditions. (a) Changes to the exit mass flux by increasing the hole area and pipe length. (b) Changes to the exit mass flux by increasing the pipe pressure and pipe length. 


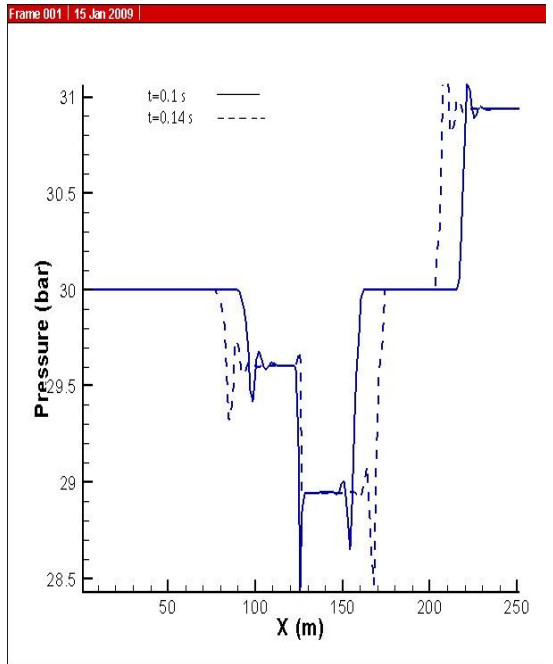

(a)

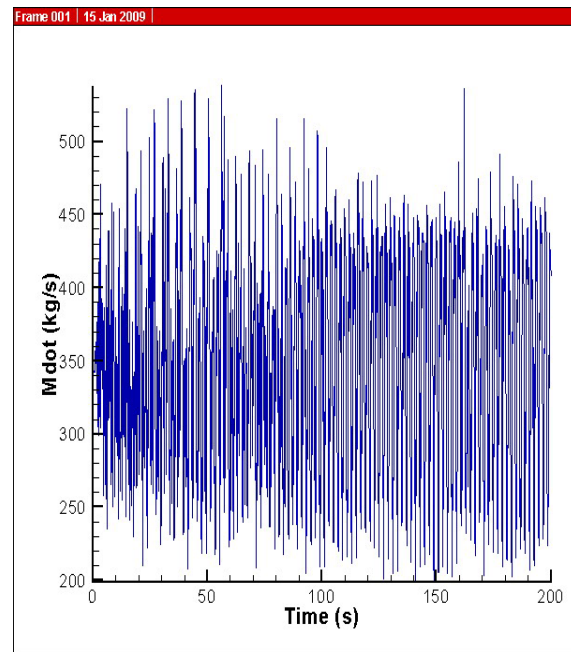

(b)

Figure 8: $\quad$ State (3) of the boundary conditions. (a) Pressure changes by increasing the pipe length at primary times. (b) Changes to the exit mass flux by time.

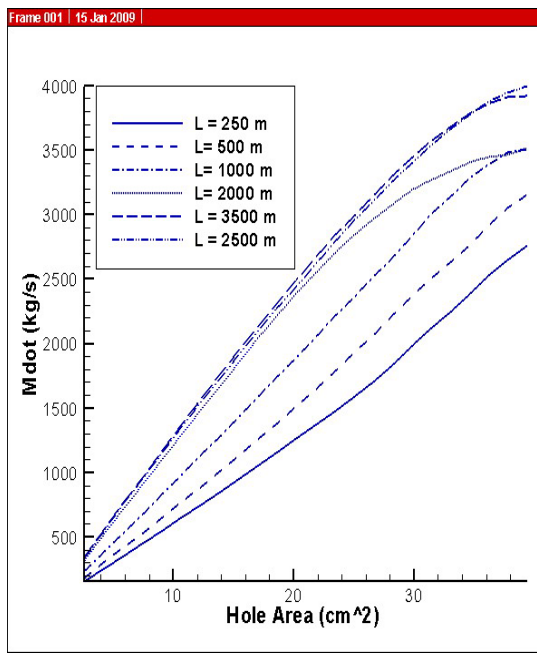

(a)

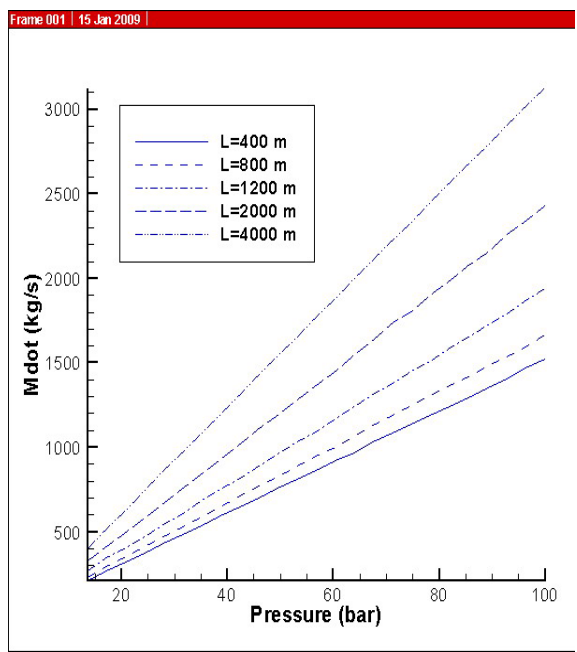

(b)

Figure 9: $\quad$ State (2) of the boundary conditions. (a) Changes to the exit mass flux by increasing the hole area and pipe length. (b) Changes to the exit mass flux by increasing the pipe pressure and pipe length. 
diagrams are rather equal. However, in the second and third states (Figure $7(\mathrm{~b})$ and 9(b)) the quantity of the exit mass flux is raised by increasing the pipe length.

\section{References}

[1] The Thermodynamics and Gas Dynamics of Internal Combustion Engines, Volume 1, Rowland S. Benson, Edited by J.H. Horlock and D.E.

Winterbone, Clarendon Press Oxford 1982.

[2] Nonsteady, One-Dimensional, Internal, Compressible Flows, JOHN A.C. KENTFIELD, Oxford University Press 1993

[3] Evaluation of gas release rate through holes in pipelines, Dong Yuhua, Gao Hilin, Zhou Jing, Feng Yaorong,2002

[4] Pipeline Design \& Construction: A Practical Approach, M. Mohitpour, H. Golshan, A. Murray, TJ930. M57 2003. 